DOI: 10.17117/na.2015.11.03.407

Поступила (Received): 23.11.2015 http://ucom.ru/doc/na.2015.11.03.407.pdf

\title{
Солопеева Ю.А.
}

\section{Оценка эффективности программно-информационных комплексов экономического назначения}

\author{
Solopeeva Yu.A. \\ Evaluation of the efficiency of software \\ programs of economic purpose
}

В данной работе рассматривается подход к созданию универсальной методики выбора операционной системы для эффективной реализации на ней программно-информационных комплексов экономического назначения

Ключевые слова: операционная система, методика, эффективность

\section{Солопеева Юлия Андреевна}

Student

Финансовый университет при правительстве РФ

2. Москва, Ленинградский проспект, 49

\begin{abstract}
In this paper, the approach to the creation of a universal method of choice of operating system for the effective implementation of economic software was considered
\end{abstract}

Key words: operating system, method, efficiency

\author{
Solopeeva Yuliya Andreevna \\ Student \\ Financial university under the government of the $R F$ \\ Moscow, Leningradsky ave., 49
}

В настоящее время на рынке программных платформ доминируют два больших семейства операционных систем - MS Windows и UNIX. Они, наряду с техническим и информационным обеспечением, определяют производительность и функциональность существующих информационно-расчетных систем, т.е. их эффективность.

Цель данной работы: произвести выбор программной платформы (операционной системы) для эффективного применения программно-информационных комплексов экономического назначения при реализации универсального и специального функционалов. При этом необходимо учитывать, что такие системы должны хранить большие базы данных и обрабатывать одновременно большое количество пользовательских запросов. Из цели вытекает объект исследования - показатели эффективности работы операционных систем.

Предметной областью исследований является совокупность существующих операционных систем.

Данная тема весьма актуальна, так как существует необходимость и отсутствие на данный момент универсальной методики выбора операционной системы для эффективной реализации на ней программно-информационных комплексов экономического назначения. Практическая значимость работы как раз 
и заключается в том, что результаты исследований позволяют выбрать подобную программную платформу (операционную систему). Научная значимость состоит в новизне способа решения известной научной задачи.

Задачи научной работы.

1. Разработка и проверка методики проведения сравнительного анализа эффективности реализации универсальных функций работы операционных систем семейств MS Windows и Linux.

2. Разработка методики проведения исследования эффективности работы программных платформ (операционных систем) при реализации специальных функций на примере межплатформенных программных комплексов Openoffice 4.x, Libreoffice 4.x, GIMP 2.8 и программного пакета для эконометрического анализа «Gretl».

Для тестирования были взяты операционные системы MS Windows 7 SP1 x86-x64, MS Windows 8.1 х86-х64, MS Windows Server 2012 R2 и Linux Ubuntu 13.10.3 x86-х64, Linux Ubuntu 12.04.4 LTS x86-х64. Данные системы в настоящее время являются наиболее современными и функциональными, а также охватывают более 85\% существующих десктопного и серверного рынков.

Все исследования проводились на двух технических платформах: AMD (MB FM2-A85XMA-E35, CPU AMD FX-8320, RAM DDR3 1600 Mgz 8 Gb) и INTEL (MB ASUS, CPU INTEL i7-4770, RAM DDR3 1600 Mgz 8 Gb) с равными (по возможности) характеристиками. Это необходимо для выявления влияния архитектуры процессоров и чипов окружения на эффективность работы программных платформ. Следует добавить, что данные архитектуры занимают 95\%-98\% рынка десктопных систем.

Каждый тест повторялся 10 раз, затем вычислялись средние значения базовых показателей.

Для решения первой задачи были проведены исследования показателей эффективности выбора программной платформы (операционной системы) при реализации универсального функционала. Для оценки эффективности значимыми базовыми метриками производительности операционной системы являются: Время загрузки операционной системы $\left(\mathrm{My}_{1}\right)$; Время выключения операционной системы (Му2); Гибернация (Муз); Пробуждение (Му4); Копирование $\mathrm{HDD} \rightarrow \mathrm{HDD}$ (Му5); Копирование HDD $\rightarrow$ USB (Му6); Копирование USB $\rightarrow$ HDD $\left(\mathrm{My7}_{7}\right.$; Копирование USB $\rightarrow$ USB (Mу8); Архивация Zip (Муя); Распаковка Zip (Му10); Архивация tar.gz (Му11); Распаковка tar.gz (Му12); Транскодирование видео (HandBrake) (My13); Кодирование в MP3 (Lame) (Mу14); Обработка изображений (RawTherapee) (My15); Процессор и память (Geekbench) (My16); Процесcop (POVRay) (Му17); Процесcop (Blender) (Му18); Установка программного комплекса (Му19); Удаление программного комплекса (Муг).

Этот базис описывает метрику пространства эффективности современных операционных систем при реализации универсального функционала.

Определим комплексный показатель эффективности выбора операционной системы при реализации универсального функционала (Му): 
$M_{y}=\frac{d_{1}}{a_{1} M_{y 1}+a_{2} M_{y 2}+a_{3} M_{y 3}+a_{4} M_{y 4}+a_{9} M_{y 9}+a_{10} M_{y 10}+\ldots+a_{15} M_{y 15}+a_{17} M_{y 17}+a_{18} M_{y 18}+a_{19} M_{y 19}+a_{20} M_{y 20}}+$ $+\mathrm{a}_{5} \mathrm{M}_{\mathrm{y} 5}+\mathrm{a}_{6} \mathrm{M}_{\mathrm{y} 6}+\mathrm{a}_{7} \mathrm{M}_{\mathrm{y} 7}+\mathrm{a}_{8} \mathrm{M}_{\mathrm{y} 8}+\mathrm{a}_{16} \mathrm{M}_{\mathrm{y} 16}$, где $\mathrm{a}_{1}, \ldots, \mathrm{a}_{20}, \mathrm{~d}_{1}$ - весовые коэффициенты.

При реализации универсального функционала наиболее эффективной оказалась операционная система Linux Ubuntu 13.10 x64 на технической платформе Intel (My= 228,0013245).

Для нахождения наиболее эффективной операционной системы, некоторые показатели необходимо минимизировать, а некоторые, наоборот - максимизировать. Для этого показатели для минимизирования заносим в знаменатель частного.

Для решения второй задачи были определены значимые базовые метрики производительности операционной системы при реализации специального функционала и проведены соответствующие тесты: Запуск программного комплекса ( $\left.\mathrm{M}_{\mathrm{C1}}\right)$; Завершение программного комплекса $\left(\mathrm{M}_{\mathrm{C} 2}\right)$; Загрузка текстового документа ( $\left.\mathrm{M}_{\mathrm{C} 3}\right)$; Редактирование текстового документа ( $\left.\mathrm{M}_{\mathrm{C} 4}\right)$; Сохранение текстового документа (Mc5); Загрузка табличного документа (Мс6); Редактирование табличного документа ( $\left.\mathrm{M}_{\mathrm{C}}\right)$; Сохранение табличного документа (MС8); Загрузка презентации ( $\left.\mathrm{M}_{\mathrm{Cg}}\right)$; Редактирование презентации ( $\left.\mathrm{M}_{\mathrm{C10}}\right)$; Сохранение презентации (Мс11); Загрузка графического файла (Мс12); Сохранение графического файла ( $\left.\mathrm{M}_{\mathrm{C13}}\right)$; Редактирование графического файла ( $\left.\mathrm{M}_{\mathrm{C14}}\right)$; Формирование тестовой базы данных и выдачи отчетов в СУБД MySQL (Mc15); Загрузка программного пакета для эконометрического анализа «Gretl» (Mc16); Сохранение программного пакета для эконометрического анализа «Gretl» (Mc17); Построение графика в программном пакете «Gretl» (Mc18); Вычисление значения функции в программном пакете «Gretl» $\left(\mathrm{M}_{\mathrm{C} 19}\right)$.

Определим комплексный показатель эффективности выбора операционной системы при реализации специального функционала $\left(\mathrm{M}_{\mathrm{c}}\right)$ : $\begin{aligned} \mathrm{M}_{\mathrm{C}}= & \frac{\mathrm{d}_{2}}{\mathrm{~b}_{1} \mathrm{M}_{\mathrm{Cl}}+\mathrm{b}_{2} \mathrm{M}_{\mathrm{C} 2}+\mathrm{b}_{3} \mathrm{M}_{\mathrm{c} 3}+\mathrm{b}_{4} \mathrm{M}_{\mathrm{c} 4}+\ldots+\mathrm{b}_{16} \mathrm{M}_{\mathrm{cl} 6}+\mathrm{b}_{17} \mathrm{M}_{\mathrm{c} 17}+\mathrm{b}_{18} \mathrm{M}_{\mathrm{c} 18}+\mathrm{b}_{19} \mathrm{M}_{\mathrm{c} 19}}, \text { где } \\ & \mathrm{b}_{1}, \ldots, \mathrm{b}_{15}, \mathrm{~d}_{2}-\text { весовые коэффициенты. }\end{aligned}$

При реализации специального функционала, самой эффективной операционной системой снова оказалась Linux Ubuntu 13.10 х64 на технической платформе Intel $\left(\mathrm{M}_{\mathrm{C}}=0,005605381\right)$.

В результате проведения исследований и применения двух разработанных выше методик, получаем комплексный показатель оценки эффективности программных платформ (операционных систем):

$\mathrm{M}=\mathrm{K}_{1} * \mathrm{M}_{\mathrm{C}}+\mathrm{K}_{2} * \mathrm{M}_{\mathrm{y}}+\mathrm{K}_{3} * \mathrm{M}_{\Phi}$, где $\mathrm{K}_{1}, \mathrm{~K}_{2}, \mathrm{~K}_{3}$ - весовые коэффициенты;

$\mathrm{M}_{\Phi}$ - показатель эффективности выбора программной платформы (OC) по функциональности (экспертная оценка), равный: $\mathrm{M}_{\Phi}=\mathrm{c}_{1}{ }^{*} \Phi-\mathrm{c}_{2}{ }^{*} \mathrm{C}, \Phi-$ функционал, C - стоимость операционной системы, с и $_{1} \mathrm{c}_{2}$ - весовые коэффициенты.

Программная платформа (операционная система) выбирается по максимальному значению комплексного показателя: $\mathrm{M} \rightarrow \max$. Итоговые показатели получились следующие: для платформы 1 (MB FM2-A85XMA-E35 , CPU AMD FX- 
8320, RAM DDR3 1600Mgz 8 Gb) - таблица 1, для платформы 2 (MB ASUS , CPU INTEL i7-4770, RAM DDR3 1600Mgz 8 GbКак) - таблица 2.

таблица 1.

\begin{tabular}{|c|c|c|c|c|c|c|c|c|c|}
\hline & $\begin{array}{l}\text { MS } \\
\text { Windows } \\
7 \text { SP1 } 1 \times 32\end{array}$ & $\begin{array}{l}\text { MS } \\
\text { Windows } \\
7 \text { SP } 1 \times 64\end{array}$ & $\left|\begin{array}{l}\text { MS } \\
\text { Windows } \\
8.1 \times 32\end{array}\right|$ & $\begin{array}{l}\text { MS } \\
\text { Windows } \\
8.1 \times 64\end{array}$ & \begin{tabular}{|l|} 
MS \\
Windows \\
Server R2 \\
x64 \\
\end{tabular} & \begin{tabular}{|l} 
Linux \\
Ubuntu \\
12.04 .4 \\
LTS x 32 \\
\end{tabular} & \begin{tabular}{|l|} 
Linux \\
Ubuntu \\
12.04 .4 \\
LTS x64 \\
\end{tabular} & \begin{tabular}{|l} 
Linux \\
Ubuntu \\
13.10 \\
x32 \\
\end{tabular} & $\begin{array}{l}\text { Linux } \\
\text { Ubuntu } \\
13.10 \times 64\end{array}$ \\
\hline$M$ & 3806,71 & 3659,2017 & 3593,635 & 3842,175 & 632,9748 & 8306,181 & 8538,972 & 8226,48 & 8506,566 \\
\hline
\end{tabular}

таблица 2.

\begin{tabular}{|l|l|l|l|l|l|l|l|l|l|}
\hline & MS & MS & MS & MS & MS & Linux & Linux & Linux & Linux \\
& Windo & Windo & Window & Window & Windows & Ubuntu & Ubuntu & Ubuntu & Ubuntu \\
& Server R2 & 12.04 .4 & 12.04 .4 & 13.10 & 13.10 \\
& Ws 7 & S $8.1 \times 32$ & S 8.1 x64 & SP1 & x64 & LTS x32 & LTS x64 & x32 & x64 \\
\hline M & 4949,9 & 4902,4 & 4764,14 & 5379,58 & 2255,911 & 9920,503 & 10246,1 & 10157 & 10533,4 \\
\hline
\end{tabular}

Исследования показали, что наиболее эффективной системой оказалась Linux Ubuntu 13.10 х64 на технической платформе Intel (M = 10533,3824).

Результаты научной работы.

1. Разработана методика проведения сравнительного анализа эффективности реализации универсальных функций работы операционных систем семейств MS Windows и Linux.

2. Разработана методика проведения исследования эффективности работы программных платформ (операционных систем) при реализации специальных функций.

3. Разработан комплексный показатель оценки эффективности программных платформ (OC) и методика его применения.

4. Проведены исследования эффективности операционных систем семейств MS Windows и Linux с применением разработанных методик. Наиболее эффективная операционная система - Linux Ubuntu 13.10 х64 (комплексный показатель равен 10533,3824).

(C) 2015, Солопеева Ю.А.

Оценка эффективности программноинформационных комплексов экономического назначения
(C) 2015, Solopeeva Yu.A.

Evaluation of the efficiency of software programs of economic purpose 